\title{
CONTROLE QUÍMICO DO MÍLDIO (Peronosclerospora sorghi) EM SORGO
}

\author{
NICÉSIO FILADELFO JANSSEN DE ALMEIDA PINTOํ, CARLOS ROBERTO CASELA², \\ ALEXANDRE DA SILVA FERREIRA ${ }^{3}$
}

${ }^{1}$ Eng. Agrônomo, Doutor em Agronomia, Pesquisador da Embrapa Milho e Sorgo, Caixa Postal 151, $35701-970$ Sete

Lagoas, MG.E-mail: nicesio@cnpms.embrapa.br (Autor para correspondência)

${ }^{2}$ Eng. Agrônomo, Ph. D., Pesquisador da Embrapa Milho e Sorgo,

${ }^{3}$ Eng. Agrônomo, M. Sc., Pesquisador da Embrapa Milho e Sorgo

Revista Brasileira de Milho e Sorgo, v.5, n.1, p.67-74, 2006

RESUMO - Sementes de sorgo da cultivar SC 283, altamente suscetível a Peronosclerospora sorghi, foram utilizadas nos três experimentos deste trabalho. Para avaliação do controle de conídios de $P$. sorghi veiculados pelas sementes, estas foram previamente tratadas com os seguintes fungicidas (g i.a.100 kg-1 sementes): metalaxyl$\mathrm{M}+$ mancozeb $(5,6+89,6)$, metalaxyl-M + chlorothalonil $(15,0+150,0)$, fosetyl-Al $(240,0)$, propamocarb $(722,0)$, metalaxyl-M $(75,0)$, metalaxyl-M $(37,5)$, fludioxonil + metalaxyl-M $(12,5+5,0)$ e fludioxonil + metalaxyl-M $(25,0+10,0)$. A seguir, as sementes foram pré-germinadas, inoculadas com conídios de $P$. sorghi e semeadas em solo esterilizado, em casa de vegetação. O delineamento experimental foi de blocos ao acaso, com nove tratamentos em quatro repetições. Os resultados evidenciaram, aos 15 dias após a semeadura, que metalaxyl-M + mancozeb, metalaxyl-M + chlorothalonil, metalaxyl-M e fludioxonil + metalaxyl-M foram altamente eficientes no controle do míldio sistêmico do sorgo ( 97,2 a 100,0\%). Para avaliação da proteção das sementes contra oosporos de $P$. sorghi presentes no solo e na palhada de sorgo, as sementes foram tratadas com os seguintes fungicidas (g i.a.100 kg-1 sementes): metalaxyl-M + mancozeb $(5,6+89,6)$, metalaxyl-M + mancozeb $(7,3+116,5)$, metalaxyl-M + chlorothalonil $(15,0$ $+150,0)$, metalaxyl-M + chlorothalonil $(19,5+195,0)$, metalaxyl-M $(37,5)$, metalaxyl$\mathrm{M}(75,0)$, metalaxyl-M + fludioxonil $(5,0+12,5)$ e metalaxyl-M + fludioxonil $(9,0$ + 22,5). O delineamento experimental foi de blocos ao acaso, com nove tratamentos em quatro repetições, sendo as parcelas estabelecidas em solo com restos de cultura de sorgo contaminados com oósporos de P. sorghi. As plantas foram avaliadas 45 dias após a semeadura, sendo que o fungicida metalaxyl-M (75,0 g i.a.100 kg-1) promoveu proteção absoluta contra a infecção sistêmica causada por oosporos de $P$. sorghi. Para estudos de controle do míldio através de pulverização foliar de fungicidas, linhas espalhadoras de inóculo de $P$. sorghi foram antecipadamente estabelecidas lateralmente às parcelas. Os seguintes fungicidas foram utilizados $\left(\mathrm{g}\right.$ i.a.ha $\left.{ }^{-1}\right)$ : metalaxyl-M + mancozeb $(100,0+$ $1600,0)$, metalaxyl-M + mancozeb $(72,0+1152,0)$, metalaxyl-M + chlorothalonil $(135,0$ $+1350,0)$, metalaxyl-M + chlorothalonil $(101,2+1012,5)$, fosetyl-Al $(2000,0)$ e fosetylAl $(1440,0)$. O delineamento foi de blocos ao acaso, com sete tratamentos em quatro repetições. As aplicações dos fungicidas, com início 21 dias após o plantio e em número de quatro, foram realizadas com pulverizador costal manual, a intervalos de 14 dias. 
Decorridos 14 dias da última pulverização, os resultados mostraram que plantas de sorgo tratadas com os fungicidas metalaxyl-M + mancozeb e metalaxyl-M + chlorothalonil não apresentaram míldio sistêmico e localizado, atendendo, assim, o padrão de lavoura (zero \%), enquanto que a testemunha sem fungicida apresentou $17,4 \%$ de plantas com míldio sistêmico e localizado.

Palavras-chave: Sorghum bicolor, fungicidas, sementes, downy mildew.

\title{
CHEMICAL CONTROL OF THE DOWNY MILDEW (Peronosclerospora sorghi) IN SORGHUM.
}

\begin{abstract}
Sorghum seeds of the cultivar SC 283, highly susceptible to Peronosclerospora sorghi were used in three experiments aiming the control of sorghum downy mildew. In the first experiment, seeds were previously treated with the following fungicides (g a. i. $100 \mathrm{Kg}^{-1}$ seeds): metalaxyl-M + mancozeb $(5.6+89.6)$, metalaxyl-M + chlorothalonil $(15.0+150.0)$, fosetyl-Al (240.0), propamocarb (722.0), metalaxyl-M (75.0), metalaxyl-M (37.5), fludioxonil + metalaxyl-M $(12.5+5.0)$ and fludioxonil + metalaxyl-M $(25.0+10.0)$. After these treatments, seeds were pregerminated, inoculated with conidia of $P$. sorghi and sowed in sterilized soil in the greenhouse. The experimental design was the one with randomized blocks, with 9 treatments in four replications. Fifteen days after sowing, the results evidenced that metalaxyl-M + mancozeb, metalaxyl$\mathrm{M}+$ chlorothalonil, metalaxyl-M and fludioxonil + metalaxyl-M were highly efficient in the control sorghum of downy mildew (97.2 to $100.0 \%$ ).

In the second experiment, fungicides were evaluated for their efficiency in giving protection to seeds against oospores of $P$. sorghi present in the soil and in sorghum debris of systemically infected plants from the previous season. The following treatments were evaluated (g a. i.100 $\mathrm{kg}^{-1}$ seeds): metalaxyl-M + mancozeb $(5.6+89.6)$, metalaxyl$\mathrm{M}+$ mancozeb $(7.3+116.5)$, metalaxyl-M + chlorothalonil $(15.0+150.0)$, metalaxyl-M + chlorothalonil $(19.5+195.0)$, metalaxyl-M (37.5), metalaxyl-M (75.0), metalaxyl-M + fludioxonil $(5.0+12.5)$ and metalaxyl-M + fludioxonil $(9.0+22.5)$. Treatments were arranged in a randomized complete block design with 9 treatments in four replications. Plots were established in soil contaminated with oospores of $P$. sorghi derived from debris of systemically infected plants in the previous season. The plants were evaluated forty-five days after the sowing, and the fungicide metalaxyl-M (75.0 g i.a.100 kg-1) showed absolute protection against the systemic infection.

The third experiment was performed to evaluate the efficiency of fungicides for the control of downy mildew through foliar application against systemic infection and local lesions of $P$. sorghi. Treatments were planted in four row-plots between two rows on a susceptible genotype, planted 40 days before, to act as spreader rows. The following fungicides were used in this experiment $\left(\mathrm{g}\right.$ i.a.ha $\left.{ }^{-1}\right)$ : metalaxyl-M + mancozeb $(100.0+$ 1600.0), metalaxyl-M + mancozeb (72.0 + 1152.0), metalaxyl-M + chlorothalonil (135.0 $+1350.0)$, metalaxyl-M + chlorothalonil (101.2 + 1012.5), fosetyl-Al (2000.0) and fosetyl$\mathrm{Al}$ (1440.0). The experimental design was a randomized block with 7 treatments in four replications. Fungicide spraying, in number of four, was accomplished with a hand sprayer at a 14 day-interval, starting 21 days after planting. Evaluations were performed fourteen
\end{abstract}


days after the last spraying and the results showed that the fungicide metalaxyl-M+ mancozeb and metalaxyl-M + chlorothalonil did not present systemically infected plants and local lesions which attends the field legislation of $0 \%$ downy mildew in seed production fields. The non-treated control had a $17.4 \%$ of systemically infected plants and local lesions.

Key words: Sorghum bicolor, fungicides, seeds, downy mildew.

O míldio do sorgo, causado pelo patógeno Peronosclerospora sorghi, é uma doença de ampla faixa de adaptação climática, sendo encontrada em várias regiões de plantio de sorgo, na África, na Ásia e nas Américas. No Brasil, a doença, antes restrita aos estados da região Sul, encontra-se atualmente disseminada em praticamente todas as áreas de plantio de sorgo. A doença pode causar perdas significativas à produção, sendo um problema principalmente em áreas de produção de sementes (Casela \& Ferreira, 2001).

Oósporos e micélio de $P$. sorghi são encontrados nas glumas, pericarpo e endosperma de sementes de sorgo coletadas de plantas infectadas sistemicamente (Safeeulla, 1976; Frederiksen, 1980).

A germinação de sementes infectadas promove alta frequiência de transmissão desse patógeno para as plântulas de sorgo. Esse fungo é estabelecido na semente sistemicamente, através da planta-mãe, ou por infecções naturais, via estigma, estilo e ovário (Pinto, 1999).

O fungicida metalaxyl é um produto sistêmico pertencente ao grupo químico alaninato, com atividade "in-vivo" e "in-vitro" contra patógenos de plantas da ordem Peronosporales, em que estão os fungos causadores de míldios em diversas culturas, como Peronosclerospora sorghi, causador do míldio do sorgo. Ele pode ser aplicado em pulverizações foliares, bem como no controle de doenças transmitidas pelo solo ou pelas sementes (Margot,
1983). Atualmente, o mercado possui esse ingrediente ativo na versão metalaxyl-M, o qual é o isômero $\mathrm{S}$ do metalaxyl anteriormente comercializado.

O tratamento de sementes de sorgo com metalaxyl a $100 \mathrm{~g}$ i.a. $100 \mathrm{~kg}^{-1}$, seguido por uma única pulverização com metalaxyl, a $750 \mathrm{~g}$ i.a.ha ${ }^{-1}$, cerca de 40 dias após o plantio, controlou completamente as infecções sistêmica e localizada causadas por $P$. sorghi. Apenas o tratamento das sementes com metalaxyl, na dose de 100-200 g i.a $100 \mathrm{~kg}^{-1}$ de sementes, não promove a proteção contra a infecção sistêmica tardia nas plantas-mãe ou perfilhos, nem contra as lesões locais sobre as folhas (Anahosur \& Patil, 1980). Em trabalho realizado no Brasil, Takeda et al. (1982) relatam que o metalaxyl (Apron $35 \mathrm{PM}$ ), aplicado em tratamento de sementes de sorgo vassoura ou de milho, nas doses de 100, 200 ou 400 g i.a. $100 \mathrm{~kg}^{-1}$ de sementes, foi o fungicida mais eficiente no controle do míldio do sorgo. Pulverizações foliares de 100 a $200 \mathrm{~g}$ i.a. $100 \mathrm{ha}^{-1}$, aplicadas 20 a 40 dias após o plantio, resultaram na completa recuperação das plantas com os sintomas da doença (Anahosur \& Patil, 1981).

De acordo com a Portaria $n^{\circ} .747$, de 24.10.1977, do Ministério da Agricultura, as sementes de sorgo devem ser produzidas em áreas onde o míldio do sorgo não tenha sido constatado. Assim, a Comissão Estadual de Sementes e Mudas - CESM/MG estabelece o Padrão de Lavoura Zero para o míldio do sorgo, em campo de produção de sementes de sorgo. 
O objetivo deste trabalho foi avaliar a eficiência de fungicidas no controle de conídios de $P$. sorghi associados às sementes de sorgo, a proteção de plantas em estádios iniciais de desenvolvimento contra os oósporos de $P$. sorghi presentes na palhada e no solo, bem como a proteção, via pulverização foliar, de plantas adultas e seus perfilhos em lavoura de produção de sementes, contra o míldio sistêmico ou localizado.

\section{Material e Métodos}

Experimento 1 - Controle de Peronosclerospora sorghi Associado às Sementes de Sorgo

Neste trabalho, conduzido em outubro de 2002, na Embrapa Milho e Sorgo, sementes de sorgo da cultivar SC 283 suscetível a $P$. sorghi, foram submetidas aos seguintes tratamentos fungicidas (g i.a.100 $\mathrm{kg}^{-1}$ de sementes): Metalaxyl-M + Mancozeb $(5,6+89,6)$, Metalaxyl-M + Chlorothalonil $(15,0+150,0)$, Fosetyl-Al (240,0), Propamocarb $(722,0)$, Metalaxyl-M (75,0), Metalaxyl-M $(37,5)$, Fludioxonil + Metalaxyl-M (12,5 + 5,0) e Fludioxonil + Metalaxyl-M $(25,0+10,0)$. Sementes sem tratamento fungicida constituíram a testemunha.

Após o tratamento com os fungicidas, as sementes foram colocadas para germinar em papel toalha umedecido, acondicionado em placas de Petri. Vinte e quatro horas após, 400 sementes germinadas, em cada tratamento, foram selecionadas, sendo 100 sementes por repetição, colocadas dentro de uma jarra de plástico de diâmetro ligeiramente superior ao da placa de Petri e altura de aproximadamente $15 \mathrm{~cm}$. Na boca da jarra, colocou-se uma tela de plástico, sobre a qual foram colocadas folhas de sorgo da cultivar suscetível SC 283 sistemicamente infectadas e com aproximadamente 18 dias de idade, com a face dorsal voltada para baixo. Sobre as folhas, colocou-se um pano úmido, o qual foi coberto com uma lâmina de plástico. $\mathrm{O}$ conjunto foi colocado a uma temperatura de 18 a $20{ }^{\circ} \mathrm{C}$, por um período de 18 horas (Casela \& Ferreira, 2001). Após esse período, as sementes foram semeadas em solo esterilizado, acondicionado em caixas de metalon $(50 \times 30 \times 10 \mathrm{~cm})$, colocadas em casa de vegetação, onde permaneceram até a época da avaliação. O delineamento experimental foi o de blocos ao acaso, com nove tratamentos em quatro repetições. Decorridos 15 dias da inoculação, as plântulas de sorgo foram avaliadas, para a verificação da presença de infecção sistêmica causada por Peronosclerospora sorghi.

Experimento 2 - Tratamento de Sementes de Sorgo Contra Oósporos de Peronosclerospora sorghi Presentes no Solo

Sementes de sorgo da cultivar SC 283 foram submetidas aos seguintes tratamentos fungicidas (g i.a.100 kg-1 de sementes): metalaxyl-M + mancozeb $(5,6+89,6)$, metalaxyl$\mathrm{M}+$ mancozeb $(7,3+116,5)$, metalaxyl-M + chlorothalonil $(15,0+150,0)$, metalaxyl-M + chlorothalonil $(19,5+195,0)$, metalaxyl-M $(37,5)$, metalaxyl-M $(75,0)$, metalaxyl-M + fludioxonil $(5,0+12,5)$ e metalaxyl-M + fludioxonil $(9,0+22,5)$. Sementes sem tratamento fungicida constituíram a testemunha. $\mathrm{O}$ delineamento experimental foi de blocos ao acaso, com nove tratamentos em quatro repetições. As sementes submetidas aos tratamentos fungicidas foram semeadas em abril de 2004, em parcelas compostas de 4 fileiras de $5 \mathrm{~m}$, espaçadas de 0,75 $\mathrm{m}$, estabelecidas em solo com restos de cultura de sorgo contaminados com oósporos de $P$. sorghi. As plantas de sorgo foram avaliadas 45 dias após a semeadura, com base nos sintomas de míldio causados por infecção sistêmica. 
Experimento 3 - Controle do Míldio do Sorgo (Peronosclerospora sorghi) com Base no Padrão de Tolerância da Doença na Lavoura de Produção de Sementes

Este experimento foi conduzido em área anteriormente cultivada com sorgo, cujas plantas apresentaram alta incidência de míldio. As parcelas experimentais foram semeadas em janeiro de 2004, com a cultivar SC 283 (4 fileiras de $5 \mathrm{~m}$ de comprimento), com sementes tratadas com metalaxyl + mancozeb $(100+1600 \mathrm{~g}$ i.a. $100 \mathrm{~kg}^{-1}$ sementes), visando proteger as sementes contra Peronosclerospora sorghi presente no solo e proteger as plantas de sorgo nos estádios iniciais de desenvolvimento. Linhas espalhadoras de inóculo de P. sorghi foram semeadas (SC 283, sem fungicida) lateralmente às parcelas, 40 dias antes da instalação do experimento. $\mathrm{O}$ delineamento foi de blocos ao acaso, com sete tratamentos em quatro repetições. Os seguintes fungicidas foram utilizados em pulverização foliar $\left(\mathrm{g}\right.$ i.a.ha $\left.{ }^{-1}\right)$ :
metalaxyl-M + mancozeb $(100,0+1600,0)$, metalaxyl-M + mancozeb $(72,0+1152,0)$, metalaxyl-M + chlorothalonil $(135,0+1350,0)$, metalaxyl-M + chlorothalonil $(101,2+1012,5)$, fosetyl-Al $(2000,0)$ e fosetyl-Al $(1440,0)$. Parcelas sem tratamento fungicida constituíram a testemunha. As aplicações dos fungicidas, com início 21 dias após o plantio e em número de quatro, foram realizadas com pulverizador costal manual, em intervalos de 14 dias. A avaliação da eficácia dos fungicidas foi realizada 14 dias após a última pulverização (77 dias após a semeadura), determinando-se a percentagem das plantas com sintoma de míldio sistêmico e lesões locais.

\section{Resultados e Discussão}

\section{Experimento 1 - Controle de Peronos-} clerospora sorghi Associado às Sementes de Sorgo

Os resultados obtidos estão apresentados na Tabela 1 e mostram que os tratamentos das

TABELA 1. Avaliação da eficiência de fungicidas no tratamento de sementes de sorgo, visando o controle de míldio causado por Peronosclerospora sorghi. Embrapa Milho e Sorgo, Sete Lagoas, MG, 2002.

\begin{tabular}{lcc}
\hline \multicolumn{1}{c}{ Tratamento } & Dose $^{\mathbf{1}}$ & Plântulas com míldio (\%) $^{\mathbf{2}}$ \\
\hline Metalaxyl-M + Mancozeb & $5,6+89,6$ & $0,25 \mathrm{c}^{3}$ \\
Metalaxyl-M + Chlorothalonil & $15,0+150,0$ & $0,00 \mathrm{c}$ \\
Fosetyl-Al & 240,0 & $48,50 \mathrm{~b}$ \\
Propamocarb & 722,0 & $75,00 \mathrm{a}$ \\
Metalaxyl-M & 75,0 & $0,25 \mathrm{c}$ \\
Metalaxyl-M & 37,5 & $1,25 \mathrm{c}$ \\
Fludioxonil + Metalaxyl-M & $12,5+5,0$ & $3,25 \mathrm{c}$ \\
Fludioxonil + Metalaxyl-M & $25,0+10,0$ & $1,25 \mathrm{c}$ \\
Testemunha sem fungicida & --------- & $82,25 \mathrm{a}$ \\
\hline C.V. (\%) & & 20,28 \\
\hline
\end{tabular}

${ }^{1}$ gramas do ingrediente ativo / $100 \mathrm{~kg}$ sementes.

${ }^{2}$ dados originais, que, para a análise de variância, foram transformados por arco seno $\sqrt{\text { Porcentagem } / 100}$. Avaliação aos 15 dias após a inoculação.

${ }^{3}$ valores seguidos pela mesma letra não diferem entre si, pelo teste de Tukey (5\%). 
sementes de sorgo com os fungicidas MetalaxylM + Mancozeb, Metalaxyl-M + Chlorothalonil, Metalaxyl-M e Fludioxonil + Metalaxyl-M foram eficientes no controle do míldio do sorgo incitado por Peronosclerospora sorghi, em sementes pré-germinadas e inoculadas com conídios deste patógeno. A eficiência do fungicida Metalaxyl no controle do míldio do sorgo encontra sustentação nas referência de Margot (1983), Anahosur \& Patil (1980, 1981), Takeda et al. (1982) e Pinto (1999). A não eficiência obtida no tratamento com o fungicida Propamocarb está de acordo com os resultados apresentados por Takeda et al. (1982). Contudo, a não eficiência do fungicida Fosetyl-Al não corrobora a citação de Pinto (1999).
Experimento 2 - Tratamento Fungicida de Sementes de Sorgo Contra Oósporos de Peronosclerospora sorghi Presentes no Solo

Os resultados apresentados na Tabela 2 mostram que o fungicida metalaxyl-M (75,0 g i.a. $100 \mathrm{~kg}^{-1}$ de sementes) difere estatisticamente em eficiência $(0,0 \%$ de míldio) em relação aos tratamentos com metalaxyl-M + mancozeb $(5,6$ $+89,6)$, metalaxyl-M + fludioxonil $(5,0+12,5)$ e da testemunha sem fungicida, porém, não difere dos demais tratamentos utilizados. A eficiência obtida com o fungicida metalaxyl no controle de Peronosclerospora sorghi está de conformidade com os relatos de Margot (1983), Takeda et al. (1982) e Pinto \& Casela (2003).

TABELA 2. Avaliação da eficiência de fungicidas no tratamento de sementes de sorgo, contra oósporos de Peronosclerospora sorghi, visando o controle do míldio do sorgo. Embrapa Milho e Sorgo, Sete Lagoas, MG, 2004.

\begin{tabular}{|c|c|c|}
\hline Tratamento & Dose $^{1}$ & $\begin{array}{l}\text { Plantas com míldio sistêmico } \\
\qquad(\%)^{2}\end{array}$ \\
\hline Metalaxyl-M + Mancozeb & $5,6+89,6$ & $1,75 b c^{3}$ \\
\hline Metalaxyl-M + Mancozeb & $7,3+116.5$ & $0,50 \mathrm{~cd}$ \\
\hline Metalaxyl-M + Chlorothalonil & $15,0+150,0$ & $0,33 \mathrm{~cd}$ \\
\hline Metalaxyl-M + Chlorothalonil & $19,5+195,0$ & $0,41 \mathrm{~cd}$ \\
\hline Metalaxyl-M & 37,5 & $0,25 \mathrm{~cd}$ \\
\hline Metalaxyl-M & 75,0 & $0,00 \mathrm{~d}$ \\
\hline Metalaxyl-M + Fludioxonil & $5,0+12,5$ & $2,50 \mathrm{ab}$ \\
\hline Metalaxyl-M + Fludioxonil & $9,0+22,5$ & $0,58 \mathrm{bcd}$ \\
\hline Testemunha sem fungicida & ---------- & $5,92 \mathrm{a}$ \\
\hline \multicolumn{2}{|l|}{ C.V. $(\%)$} & 31,82 \\
\hline \multicolumn{3}{|c|}{$\begin{array}{l}{ }^{1} \text { gramas do ingrediente ativo } 100 \mathrm{~kg}^{-1} \text { sementes. } \\
{ }^{2} \text { dados originais, que, para a análise de variância, foram transformados por por } \sqrt{\text { Porc }} \\
\text { a semeadura. } \\
{ }^{3} \text { valores seguidos pela mesma letra não diferem entre si, pelo teste de Tukey }(5 \%) \text {. }\end{array}$} \\
\hline
\end{tabular}


Experimento 3 - Controle do Míldio do chlorothalonil não apresentaram plantas com Sorgo (Peronosclerospora sorghi), com Base no míldio sistêmico e lesões localizadas, atendendo Padrão de Tolerância da Doença na Lavoura o padrão de lavoura (zero \%), enquanto a testede Produção de Sementes

Os resultados apresentados na Tabela 3 mostraram que os tratamentos fungicidas metalaxyl-M + mancozeb e metalaxyl-M + munha apresentou $17,4 \%$ de plantas com míldio sistêmico e lesões localizadas. Estes resultados estão de acordo com aqueles apresentados por Anahosur \& Patil (1980, 1981).

TABELA 3. Avaliação da eficiência de fungicidas foliares no controle do míldio (Peronosclerospora sorghi) em sorgo. Embrapa Milho e Sorgo, Sete Lagoas, MG, 2004.

\begin{tabular}{|c|c|c|c|}
\hline Tratamento & \multicolumn{2}{|c|}{ Dose $^{1}$} & Plantas com míldio ${ }^{2}(\%)$ \\
\hline Metalaxyl-M + Mancozeb & \multicolumn{2}{|c|}{$100,0+1600,0$} & $0,0 \mathrm{c}^{3}$ \\
\hline Metalaxyl-M + Mancozeb & \multicolumn{2}{|c|}{$72,0+1152,0$} & $0,0 \mathrm{c}$ \\
\hline Metalaxyl-M + Chlorothalonil & \multicolumn{2}{|c|}{$135,0+1350,0$} & $0,0 \mathrm{c}$ \\
\hline Metalaxyl-M + Chlorothalonil & \multicolumn{2}{|c|}{$101,2+1012,5$} & $0,0 \mathrm{c}$ \\
\hline Fosetyl-Al & \multicolumn{2}{|c|}{2000,0} & $3,6 \mathrm{~b}$ \\
\hline Fosetyl-Al & \multicolumn{2}{|c|}{1440,0} & $4,1 \mathrm{~b}$ \\
\hline Testemunha sem fungicida & \multicolumn{2}{|c|}{--------- } & $17,4 \mathrm{a}$ \\
\hline C.V. $(\%)$ & & & 17,07 \\
\hline \multicolumn{4}{|c|}{$\begin{array}{l}{ }^{1} \text { gramas do ingrediente ativo / ha } \\
{ }^{2} \text { dados originais, que, para a análise de variância, foram transformados por } \sqrt{\text { Porcent }} \\
\text { semeadura. } \\
{ }^{3} \text { valores seguidos pela mesma letra não diferem entre si, pelo teste de Tukey }(5 \%) \text {. }\end{array}$} \\
\hline $\begin{array}{l}\text { Conclusões } \\
\text { 1-Os fungicidas metalaxyl-M+ } \\
\text { metalaxyl-M + chlorothalonil, meta } \\
\text { fludioxonil + metalaxyl-M, nas doses ut } \\
\text { tratamento das sementes, são eficientes } \\
\text { do míldio do sorgo causado por Peronos } \\
\text { sorghi, em sementes pré-germinadas e } \\
\text { com conídios desse patógeno; } \\
2 \text { - O fungicida metalaxyl-M, } \\
75,0 \text { g i.a. } 100 \mathrm{~kg}^{-1} \text { de sementes, prom } \\
\text { ção absoluta contra a infecção sistêmic } \\
\text { por oósporos de } P \text {. sorghi presentes } \\
\text { de sorgo e no solo; }\end{array}$ & $\begin{array}{l}\text { ncozeb, } \\
\text { yl-M e } \\
\text { adas no } \\
\text { controle } \\
\text { rospora } \\
\text { culadas } \\
\text { dose de } \\
\text { prote- } \\
\text { ausada } \\
\text { alhada }\end{array}$ & $\begin{array}{l}\text { Campc } \\
\text { Verneq } \\
\text { Milho } \\
\text { (Operá } \\
\text { dução c }\end{array}$ & $\begin{array}{l}\text { ossível produzir sementes de sorgo } \\
\text { ivre de míldio (Peronosclerospora } \\
\text { ante pulverizações foliares preven- } \\
\text { ungicidas metalaxyl-M + mancozeb } \\
\text { M + chlorothalonil. } \\
\text { Agradecimentos } \\
\text { ni Alves da Silva, José Moreira } \\
\text { óvis Geraldo Ribeiro, Ademar } \\
\text { sistentes de Operações da Embrapa } \\
\text { go) e Gilberto Ribeiro Rodrigues } \\
\text { ral), a valiosa colaboração na con- } \\
\text { rabalho. }\end{array}$ \\
\hline
\end{tabular}




\section{Literatura Citada}

ANAHOSUR, K. N.; PATIL, S. H. Chemical control of sorghum downy mildew in India. Plant Disease, St. Paul, v. 64, p. 1004-1006, 1980.

ANAHOSUR, K. N.; PATIL, S. H. Effect of metalaxyl seed treatment on seedling emergence and development of local lesions of sorghum downy mildew in sorghum. Newsletter of the International Working Group on Graminaceous Downy Mildews. Patancheru, ICRISAT, III, 1, 1981.

CASELA, C. R.; FERREIRA, A. S. O Míldio do Sorgo. Sete Lagoas: EMBRAPA-CNPMS, 2001. 7 p. (EMBRAPA-CNPMS. Circular Técnica, 12).

FREDERIKSEN, R. A. Sorghum downy mildew in the United States: overview and outlook. Plant Disease, St. Paul, v. 64, p. 903-908, 1980.

MARGOT, P. Control of seed-borne diseases with metalaxyl. Seed Science \& Technology, Zurich, v. 11, n. 3, p. 921-933, 1983.
PINTO, N. F. J. A. Patologia de Sementes de Sorgo. Sete Lagoas: EMBRAPA-CNPMS, 1999. 62 p. (EMBRAPA-CNPMS, Circular Técnica, 32).

PINTO, N. F. J. A.; CASELA, C. R. Eficiência de fungicidas no controle do míldio do sorgo (Peronosclerospora sorghi) associado às sementes de sorgo. Informativo Abrates, Brasília, DF, v. 13, n. 3, p. 225, 2003.

SAFEEULLA, K. M. Biology and control of downy mildews of pearl millet, sorghum, and finger millet. Mysore: Wesley Press, 1976. $304 \mathrm{p}$.

TAKEDA, A. S.; NAKAMURA, K.; GIMENES-FERNANDES, N.; KRONKA, S. N.; GOMES, G.; INOUE, L. T. Efeito do tratamento químico de sementes sobre o controle do míldio do sorgo em milho e em sorgo. Científica, Jaboticabal, v. 10, n. 1, p. 129$133,1982$. 